\title{
The new era of precision medicine in hepatocellular carcinoma: the urgent need for promising biomarkers
}

\author{
Haruhiko Takeda ${ }^{1,2}$, Hiroki Nishikawa ${ }^{2,3}$, Yukio Osaki ${ }^{2,4}$ \\ ${ }^{1}$ Department of Gastroenterology and Hepatology, Graduate School of Medicine, Kyoto University, Kyoto, Japan; ${ }^{2}$ Department of Gastroenterology \\ and Hepatology, Osaka Red Cross Hospital, Osaka, Japan; ${ }^{3}$ Department of Gastroenterology and Hepatology, Hyogo College of Medicine, \\ Nishinomiya, Japan; ${ }^{4}$ Department of Gastroenterology and Hepatology, Meiwa Hospital, Nishinomiya, Japan \\ Correspondence to: Haruhiko Takeda, MD, PhD. Department of Gastroenterology and Hepatology, Graduate School of Medicine, Kyoto University, \\ 54 Shogoin-Kawahara-cho, Sakyo-ku, Kyoto 606-8507, Japan. Email: htakeda@kuhp.kyoto-u.ac.jp. \\ Comment on: Kudo M, Finn RS, Qin S, et al. Lenvatinib versus sorafenib in first-line treatment of patients with unresectable hepatocellular \\ carcinoma: a randomised phase 3 non-inferiority trial. Lancet 2018;391:1163-73.
}

Submitted Aug 15, 2018. Accepted for publication Aug 20, 2018.

doi: 10.21037/hbsn.2018.08.06

View this article at: http://dx.doi.org/10.21037/hbsn.2018.08.06

Hepatocellular carcinoma (HCC) is the second most lethal malignancy worldwide, and the prognosis of advanced HCC is especially dismal. After the SHARP trial reported in 2007, a number of phase III trials of molecular targeting agents for advanced HCC have been conducted, but most of them have failed to achieve their primary endpoint, and sorafenib has been the only first-line systemic therapy approved for HCC in this decade (1).

In 2018, Kudo et al. reported a multicenter, randomized, open-label, phase III trial (REFLECT trial), in which lenvatinib compared with sorafenib, demonstrated a treatment effect on overall survival (OS) by statistical confirmation of non-inferiority (2). Lenvatinib is an oral multi-kinase inhibitor targeting kinases including fibroblast growth factor receptor (FGFR) 1-4, vascular endothelial growth factor receptor (VEGFR) 1-3, plateletderived growth factor receptor alpha (PDGFR $\alpha$ ), RET and KIT (1). This novel anti-angiogenesis multi-kinase inhibitor has recently been introduced into clinical practice as one of the systemic chemotherapy regimens for advanced HCC, along with sorafenib.

The strength of the REFLECT trial is not only its non-inferiority to sorafenib in terms of OS as the primary endpoint, but also superiority to sorafenib in terms of progression-free survival, time to progression, and objective response rate (ORR) as secondary endpoints. In addition, the major lenvatinib-related adverse events, including hypertension and proteinuria, are not life-threatening and do not have significant impact on quality of life. This evidence suggests that lenvatinib may be an option for firstline systemic therapy in patients with unresectable HCC.

The high ORR of lenvatinib reflects the antiangiogenesis effect and intratumor necrotic change. Furthermore, recently reported combination therapy with lenvatinib and pembrolizumab, a novel anti-PD-L1 antibody, demonstrated more than $40 \%$ of ORR (3), compatible with that of conventional trans-arterial catheter chemoembolization (TACE) (4). These anti-tumor effects can be considered as strong as conventional TACE, suggesting that molecular targeted therapy would be the main treatment option for various stages of HCC patients including both BCLC-B and BCLC-C cases.

In the near future, several additional novel molecular targeted agents are expected to be approved including antiprogrammed death (PD)-1 or anti-PD-L1 antibodies. This will provide us with the means needed to establish some biomarkers helpful for choosing the appropriate regimen for each patient. To date, in the treatment guidelines of several cancers, personalized medicine using several genetic biomarkers has been approved in clinical practice. For example, in lung cancer, point mutation of EGFR or EML4-ALK fusion have been detected to be significant genetic biomarkers to select particular molecular targeted agents. Thus, in the clinical setting, clinicians generally perform tumor biopsy to examine biomarkers and then select the appropriate treatment regimen for each patient. In addition, microsatellite instability-high has been discussed as a novel biomarker to use with recently 
developed immuno-checkpoint inhibitors, and in 2017, the FDA approved pembrolizumab, a novel PD-1 inhibitor, for patients with microsatellite instability-high or mismatchrepair-deficient solid tumors, regardless of tumor site.

Although there have been no established biomarkers in molecular targeted therapy for advanced HCC, recently several trials have been conducted investigating the biomarkers predicting the responders of sorafenib monotherapy using liver tumor samples. Arao et al. reported that focal amplification of FGFR3/4 is associated with responders of sorafenib using the comparative genomic hybridization $(\mathrm{CGH})$ array and real-time polymerase chain reaction-based copy number assay (5). We performed targeted sequencing of HCC-related genes using a formalin-fixed paraffin-embedded (FFPE) sample of fine needle liver biopsy (6). Despite the degradation of DNA and RNA from clinical FFPE samples, targeted DNA and RNA sequencing could be successfully performed and sufficient sequence data could be generated. From RNA sequencing results, higher expression of $N R G 1$, a ligand of EGFR family, was revealed to correlate with poorer progression free survival time in HCC patients treated with sorafenib. The fact that clinical FFPE samples, rather than frozen tumor tissues, can be used for genetic analysis should be emphasized in considering the possibilities of introduction of genomic biomarkers to real-world clinical practice.

On the other hand, there have been several reports of tumor heterogeneity in various cancers including HCC. In particular, the nature of HCC is multi-focal carcinogenesis from cirrhotic liver, and every tumor in the same cirrhotic liver has a different genetic landscape. This means that the result of genetic analysis can vary based on, not only from which tumor, but also from where in the tumor, fine needle biopsy is performed. One of the strategies expected to overcome these issues is so-called "liquid biopsy". Cell free DNA or circulating tumor cells in the blood flow can reflect the overall genetic or transcriptional profiles of all tumors existing in a patient. Several studies on genetic analysis of cell free DNA or circulating tumor cells have suggested the usefulness of these liquid biopsies as possible biomarkers to predict the anti-tumor efficacy or survival time, although they have not been adequately validated to date (7).

In conclusion, approval of lenvatinib opened the new era of molecular targeting therapy for HCC. It requires the use of several molecular targeted agents appropriate for each HCC patient. To realize this personalized medicine, the establishment of genetic or transcriptional biomarkers needed to select the appropriate regimen is eagerly awaited.

\section{Acknowledgements}

None.

\section{Footnote}

Conflicts of Interest: The authors have no conflicts of interest to declare.

\section{References}

1. Kudo M. A New Era of Systemic Therapy for Hepatocellular Carcinoma with Regorafenib and Lenvatinib. Liver Cancer 2017;6:177-84.

2. Kudo M, Finn RS, Qin S, et al. Lenvatinib versus sorafenib in first-line treatment of patients with unresectable hepatocellular carcinoma: a randomised phase 3 noninferiority trial. Lancet 2018;391:1163-73.

3. Ikeda $M$, Kudo $M$, Kobayashi $M$, et al. A phase $1 \mathrm{~b}$ trial of lenvatinib (LEN) plus pembrolizumab (PEM) in patients (pts) with unresectable hepatocellular carcinoma (uHCC). J Clin Oncol 2018;36:abstr 4076.

4. Peck-Radosavljevic M, Kudo M, Raoul JL, et al. Outcomes of patients (pts) with hepatocellular carcinoma (HCC) treated with transarterial chemoembolization (TACE): Global OPTIMIS final analysis. J Clin Oncol 2018;36:abstr 4018.

5. Arao T, Ueshima K, Matsumoto K, et al. FGF3/FGF4 amplification and multiple lung metastases in responders to sorafenib in hepatocellular carcinoma. Hepatology 2013;57:1407-15.

6. Sakai K, Takeda H, Nishijima N, et al. Targeted DNA and RNA sequencing of fine-needle biopsy FFPE specimens in patients with unresectable hepatocellular carcinoma treated with sorafenib. Oncotarget 2015;6:21636-44.

7. Liao $\mathrm{W}$, Yang $\mathrm{H}, \mathrm{Xu} \mathrm{H}$, et al. Noninvasive detection of tumor-associated mutations from circulating cell-free DNA in hepatocellular carcinoma patients by targeted deep sequencing. Oncotarget 2016;7:40481-90.

Cite this article as: Takeda $\mathrm{H}$, Nishikawa $\mathrm{H}$, Osaki Y. The new era of precision medicine in hepatocellular carcinoma: the urgent need for promising biomarkers. HepatoBiliary Surg Nutr 2018;7(6):490-491. doi: 10.21037/hbsn.2018.08.06 